\title{
Ungueal melanoma and a generalized vitiligo: Is this a rare association?
}

\author{
Hanane Daflaoui', Loubab Omahsan', Adnane Lachkar², Abdelouahed Najib², \\ Siham Dikhaye ${ }^{1,3}$, Nada Zizi ${ }^{1,3}$
}

${ }^{1}$ Department of Dermatology, Mohammed VI University Hospital, Medical School of Oujda, Mohammed First University, Oujda, Morocco, ${ }^{2}$ Department of Traumatology B, Mohammed VI University Hospital, Medical School of Oujda, Mohammed First University, Oujda, Morocco, ${ }^{3}$ Laboratory of Epidemiology, Clinical research and Public Health, Medical School of Oujda, Mohammed First University of Oujda, Morocco

Corresponding author: Dr. Hanane Daflaoui, E-mail: dr.hanane.daf@gmail.com

\begin{abstract}
We report an observation of a good prognosis ungual melanoma showed up 10 years after the onset of a generalized vitiligo. It's a 66ans years old male, with a history of a rapidly progressing vulgar vitiligo showed up 10 years earlier. The patient has been referred to us for the management of a nail melanoma, retained on clinical and histological data, with a vitiligo extended to 70\% Body Surface Area. The assessment of locoregional and distant extension was without abnormalities. Several authors have reported the association of vitiligo and other autoimmune disorders, nonmelanoma skin cancers, or as part of a particular syndrome. The association of ungueal melanoma and generalized vitiligo was rarely reported in literature. Across this observation, we illustrate that The association of cutaneous melanoma and vitiligo is not rare and may be a factor of good prognosis.
\end{abstract}

Key words: Vitiligo; Melanoma; Ungueal melanoma

\section{INTRODUCTION}

Vitiligo is an autoimmune skin disease which is manifested by achromic patches, due to destruction of melanocytes. We report through this observation a case of a good prognosis ungual melanoma showed up 10 years after the onset of a generalized vitiligo.

\section{CASE REPORT}

It's a 66ans years old male, with a history of wellbalanced diabetes and rapidly progressing vulgar vitiligo showed up 10 years earlier. The patient has been referred to us for the management of a nail melanoma, retained on clinical and histological data, with a Breslow thickness at $3 \mathrm{~mm}$ (Fig. 1). Clinical examination had found a conscious patient, well oriented in time and space, with a vitiligo extended to $70 \%$ Body Surface Area (Fig. 2).
Dermatological examination also identified a dark crust in the outer portion of the right thumbnail and in the lateral nail fold associated with paronychia, without any transit lesions, no palpable lymphadenopathy was apparent (Fig. 3).

Dermoscopy has showed crusts and scals with the destruction of the lateral nail fold, with lack of the chaos aspect and no atypical vascularization (Fig. 4).

The assessment of locoregional and distant extension was without abnormalities, including the ultrasound of the right upper limb lymph nodes, cerebral and thoracoabdominal-pelvic scan and also a pet-scann. The excision of the tumor was performed with margins of $2 \mathrm{~cm}$.

\section{DISCUSSION}

Ungueal melanoma is an infrequent disease. Usually overlooked by the patient and diagnosed at late stages.

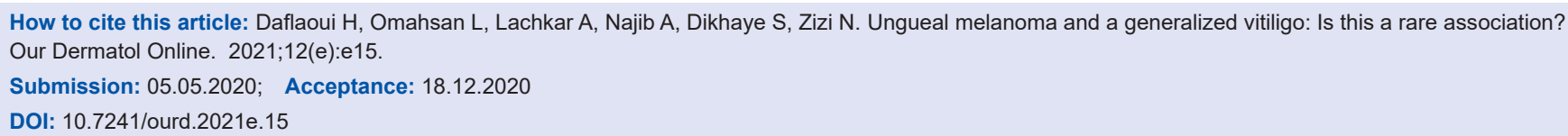




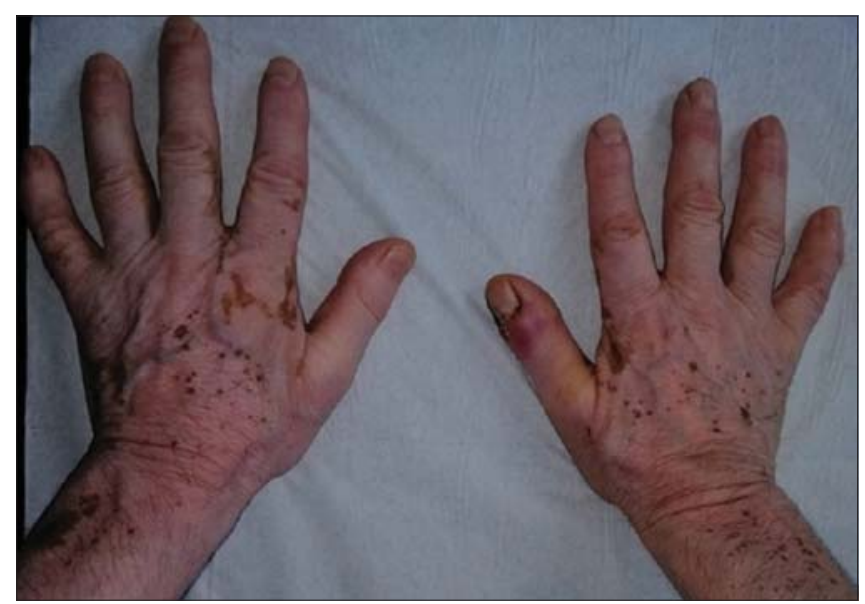

Figure 1: Clinical examination has shown large hypopigmented patches extended to $70 \%$ Body Surface Area, the Vitiligo Extent Score (VES): 42.96.

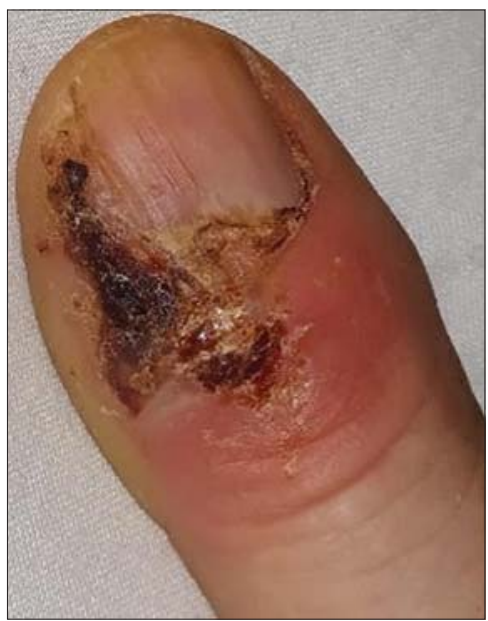

Figure 2: A distal longitudinal melanonychia in the center of the nail of the $5^{\text {th }}$ left finger.

Vitiligo is a very common autoimmune disease, it affects $2 \%$ of general population [1].

Several authors have reported the association of vitiligo and other autoimmune disorders, non-melanoma skin cancers, or as part of a particular syndrome [2].

The association «vitiligo» and «cutaneous melanoma» was initially considered as rare. Later, it turned out that the occurrence of melanoma in patient with vitiligo was more frequent than general population [2].

In a related German study that evaluated 623 patients with cutaneous melanoma, $3.7 \%$ had vitiligo [3]. It can be diagnosed before or after the diagnosis of melanoma.

For more than 40 years, many published studies have shown that patients with both melanoma and vitiligo have better prognosis and a longer lifetime [4].

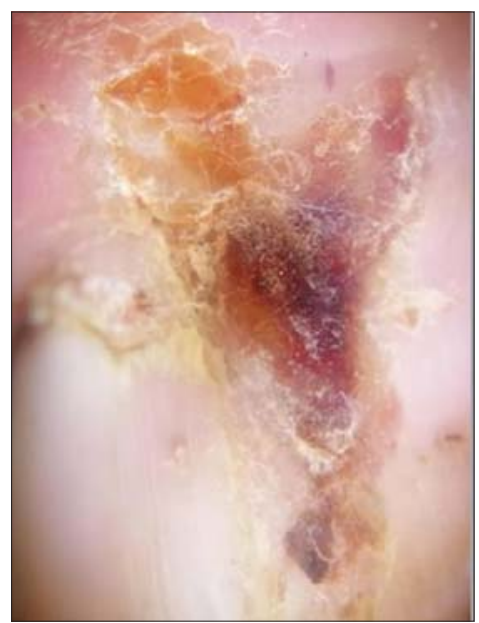

Figure 3: Dermoscopy has showed crusts and scals with the destruction of the lateral nail fold, with lack of the chaos aspect and no atypical vascularization.

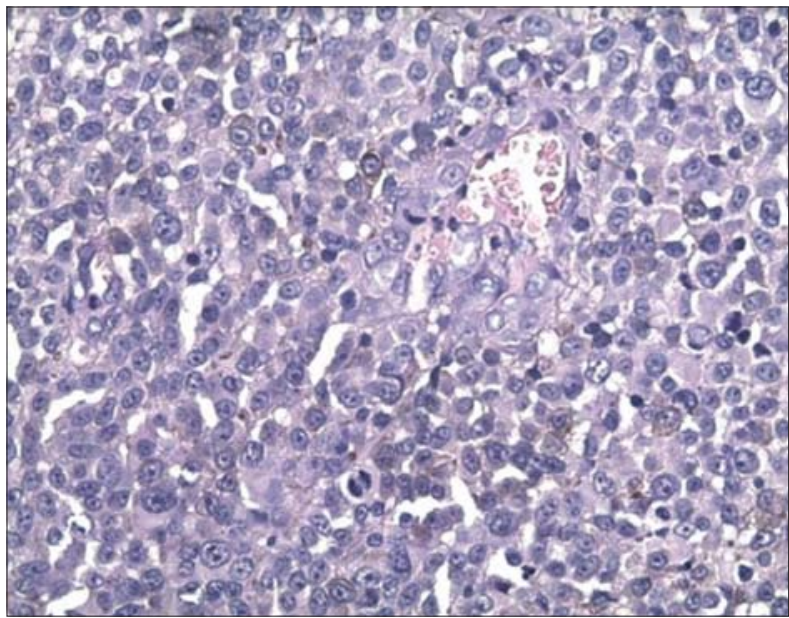

Figure 4: A pagetoid migration of isolated cells and a migration through the nail tablette of melanic pigment and tumor cells.

The association of ungueal melanoma and generalized vitiligo was rarely reported in literature [5].

\section{CONCLUSION}

Across this observation, we illustrate that The association of cutaneous melanoma and vitiligo is not rare and may be a factor of good prognosis. It association with ungueal melanoma is not quite reported.

\section{Consent}

The examination of the patient was conducted according to the Declaration of Helsinki principles.

The authors certify that they have obtained all appropriate patient consent forms. In the form the patient(s) has/have given his/her/ their consent for his/her/their images and other clinical information 
www.odermatol.com

to be reported in the journal. The patients understand that their names and initials will not be published and due efforts will be made to conceal their identity, but anonymity cannot be guaranteed.

\section{REFERENCES}

1. Failla CM, Carbone ML, Fortes C, Pagnanelli G, D'Atri S. Melanoma and vitiligo: in good company. Int J Mol Sci. 2019;20:5731.

2. Alikhan A, Felsten LM, Daly M, Petronic-Rosic V. Vitiligo: A comprehensive overview. JAAD. 2011;65:473-91.

3. Schallreuter KU, Levenig C, Berger J. Vitiligo and cutaneous melanoma. A case study. Dermatologica. 1991;183:239-45.

4. Cohen BE, Manga P, Lin K, Elbuluk N. Vitiligo and melanoma associated vitiligo : understanding their similarities and differences. Am J Clin Dermatol. 2020;21:669-80.

5. Oliveira CIC. Ungueal melanoma and vitiligo: case report. Medicina (Ribeirão Preto). 2015;48:512-7.

Copyright by Hanane Daflaoui, et al. This is an open access article distributed under the terms of the Creative Commons Attribution License, which permits unrestricted use, distribution, and reproduction in any medium, provided the original author and source are credited.

Source of Support: Nil, Conflict of Interest: None declared. 